Sección: Casos clínicos, médicos o quirúrgicos

Lesión dermo-hemorrágica en un canino compatible con loxoscelismo. Relato de un caso

Artículo de Hernández D, Ruiz C, Marega N, Bruni, M

CIENCIA VETERINARIA, Vol. 24, № 1, enero-junio de 2022, ISSN 1515-1883 (impreso) E-ISSN 1853-8495 (en línea)

DOI: http://dx.doi.org/10.19137/cienvet202224109

\title{
Lesión dermo-hemorrágica en un canino compatible con loxoscelismo. Relato de un caso
}

\section{Dermo-hemorrhagic lesión in a canine compatible with loxoscelism. Case report}

\section{Lesão dermo-hemorrágica em um canino compatível com loxoscelismo. Relato de um caso}

\author{
Hernández $D^{1}$, Ruiz $C^{1}$, Marega $N^{1}$, Bruni, $M^{1}$ \\ 1 Facultad de Ciencias Veterinarias, Universidad Nacional de La Pampa, calle 5 esq. 116, General Pico \\ (6360), La Pampa. Republica Argentina
}

Correo electrónico:mbruni@vet.unlpam.edu.ar

Fecha de recepción: 07/07/2021

Fecha de aceptación para su publicación: 20/09/2021

\section{RESUMEN}

Los accidentes provocados por arañas del género Loxosceles son importantes en Medicina Veterinaria por la gravedad de la lesión que ocasionan y las posibles complicaciones sistémicas que pueden llevar a la muerte del animal. Sin embargo, existen pocos registros epidemiológicos. Por ello surge la necesidad de difundir los conocimientos básicos para pensar en loxoscelismo ante un cuadro compatible, y para realizar un diagnóstico temprano a partir de los datos clínicos y la anamnesis, con la identificación del animal causante del accidente cuando fuese posible. El presente trabajo tiene como objetivo describir la evolución de las lesiones probablemente causadas por envenenamiento de Loxosceles sp. en un canino hembra, raza Fox Terrier, de 6 años, $7 \mathrm{~kg}$, atendida en el servicio de Guardia del Hospital Escuela de Animales Pequeños de la Facultad de Ciencias Veterinarias UNLPam. Se observaron lesiones en la conjuntiva ocular izquierda, con sangrado evidente, labio superior del mismo lado con franca edematización y 
hematoma también con sangrado; edema y hematomas en miembros posteriores en la región poplítea y del muslo. Los resultados de laboratorio evidenciaron signos de anemia hemolítica y coagulopatía. El animal fue tratado con antiveneno específico, transfusión de sangre entera fresca, corticoides y antibióticos. Por los datos clínicos y las evidencias de la presencia de Loxosceles sp. en el domicilio (ootecas y mudas), se conjetura que el envenenamiento canino sería un caso de loxoscelismo dermo-hemolítico, con evolución favorable del paciente al cabo de una semana a pesar de la gravedad del mismo.

Palabras clave Loxosceles, canino, coagulopatía, envenenamiento, tratamiento

\section{ABSTRACT}

Accidents caused by Loxosceles spiders are important in Veterinary Medicine due to the severity of the injury they cause and the possible systemic complications that can lead to the animal's death. However, there are few epidemiological records. This is why it is necessary to generate basic knowledge to consider loxoscelism as a possible condition, to generate an early diagnosis from clinical data and anamnesis, with the identification of the animal that caused the accident when it is possible. The present study aims to describe the evolution of the lesions probably caused by Loxosceles sp. poisoning in a 6-year-old female canine, $7 \mathrm{~kg}$, Fox Terrier breed, received at the Small Animal School Hospital of Veterinary Sciences Faculty, UNLPam. Lesions were observed on the left ocular conjunctiva, with evident bleeding, left upper lip with swelling and hematoma also with bleeding; edema and bruising of hind limbs in the popliteal and thigh regions. Laboratory studies showed hemolytic anemia and coagulopathy signs. The animal was treated with specific antivenom, fresh whole blood transfusion, corticosteroids, and antibiotics. Based on the clinical data and the evidence of the presence of Loxosceles sp. in the house (ootheca and molts), it is conjectured that this could be a case of dermo-hemolytic loxoscelism, with favorable evolution of the patient one week later, despite the severity of the case.

Keywords: Loxosceles sp., canine, coagulopathy, poisoning, treatment 


\section{RESUMO}

Os acidentes causados por aranhas do gênero Loxosceles são importantes na Medicina Veterinária pela gravidade das lesões que causam e pelas possíveis complicações sistêmicas que podem levar à morte do animal. No entanto, existem poucos registros epidemiológicos. Por isso, surge a necessidade de disseminar os conhecimentos básicos para pensar em loxoscelismo diante de um quadro compatível e para realizar um diagnóstico precoce com base em dados clínicos e anamnese, com a identificação do animal causador do acidente quando possível. 0 presente trabalho tem como objetivo descrever a evolução das lesões provavelmente causadas por envenenamento por Loxosceles $s p$. em um canino fêmea da raça Fox Terrier, 6 anos, $7 \mathrm{~kg}$, atendida no Serviço de Emergência do Hospital Escola de Pequenos Animais da Faculdade de Ciências Veterinárias da UNLPam. As lesões foram observadas na conjuntiva ocular esquerda, com sangramento evidente, lábio superior do mesmo lado com edema acentuado e hematoma também com sangramento; edema e hematomas dos membros posteriores na região poplítea e da coxa. Os resultados laboratoriais mostraram sinais de anemia hemolítica e coagulopatia. 0 animal foi tratado com antiveneno específico, transfusão de sangue total fresco, corticoides e antibióticos. Pelos dados clínicos e as evidências da presença de Loxosceles sp. no domicílio (ootecas e mudas), conjetura-se que o envenenamento canino seria um caso de loxoscelismo dermo-hemolítico, com evolução favorável do paciente após uma semana, apesar de sua gravidade.

Palavras-chave: Loxosceles, canino, coagulopatia, envenenamento, tratamento.

\section{Introducción}

En Argentina existen 5 especies del género Loxosceles que pertenecen a la familia Sicariidae ${ }^{(1)}$. La especie Loxosceles laeta es considerada como la más peligrosa porque es capaz de producir la muerte al ser humano. Ocupa a nivel nacional y mundial los primeros lugares en cuanto a cantidad de accidentes y peligrosidad ${ }^{(2)}$. En Medicina Veterinaria también es de importancia sanitaria por las lesiones que genera y las posibles complicaciones sistémicas que pueden ocasionarle la muerte al animal. Sin embargo, en el campo de la clínica veterinaria, existen pocos reportes epidemiológicos. Se considera inexistente en animales 
grandes, ya que, dado el grosor de la piel, esta no es perforable, rara en gatos y más común en perros, particularmente por su hábito explorador y curioso ${ }^{(3)}$.

Habitualmente es conocida como "araña marrón", “araña de los rincones" o "araña de los cuadros" y su tamaño varía entre pocos $\mathrm{mm}$ a $3-4 \mathrm{~cm}$, incluyendo sus patas. En su cefalotórax tiene una depresión con un aspecto parecido a un "violín" (de allí su nombre). Posee 3 pares de ojos ubicados en forma de "V", muy útiles para su diferenciación con otras especies no peligrosas (Foto 1). Posee hábitos domiciliarios, habita lugares secos y oscuros, detrás de muebles, cuadros, en cielorrasos de madera, hendiduras de paredes, en depósitos y ropas guardadas. Su temperamento no es agresivo, generalmente no pica, excepto cuando es apretada o aprisionada contra el cuerpo, por encontrarse en la vestimenta o en el calzado ${ }^{(4)}$.

La toxicidad del veneno se debe a la presencia, entre otras, de la esfingomielinasa, una proteína de $35 \mathrm{kDa}$ llamada F35, que sería la de mayor importancia. Sus isoformas P1 y P2 poseen actividad de esfingomielinasa - D que "in vivo" causan hemólisis y dermonecrosis ${ }^{(2)}$. El cuadro clínico se conoce con el nombre de loxoscelismo con dos manifestaciones, que no necesariamente se suceden una luego de la otra: cutánea o local, la forma más frecuente, y visceral o sistémica, menos común pero la forma más grave de presentación. En el loxoscelismo cutáneo, luego de la picadura, que en un principio puede pasar desapercibida, aparece dolor y edema. Se forma una pápula que puede evolucionar a una placa eritematosa o adoptar las características de placa "livedoide" o marmórea (áreas equimóticas que alternan con áreas pálidas isquémicas brindando un aspecto marmolado). La lesión se rodea de un halo claro, quedando bien delimitado, en las primeras 24 - 48 horas $^{(5)}$. La placa se transforma en escara a la segunda o tercera semana. Puede acompañarse por petequias, ampollas, prurito y vómitos. El loxoscelismo sistémico suele desencadenarse entre las 6 a 24 horas de ocurrido el accidente. Produce hemólisis intravascular, anemia hemolítica, hipertermia, hematuria, hemoglobinuria, ictericia, coagulación intravascular diseminada (CID) e insuficiencia renal aguda y posible muerte. La plaquetopenia constituye el primer indicio de progresión a la CID ${ }^{(6)}$.

El diagnóstico de una picadura de Loxosceles sp. debe basarse en el cuadro clínico del paciente. Los análisis complementarios inespecíficos que se realicen contribuyen al seguimiento ${ }^{(7)}$. El examen clínico debe ir acompañado de una anamnesis detallada, con la cronología de los signos y síntomas que aparezcan. La captura e identificación del 
animal o indicadores de su presencia (ootecas, mudas) son de suma importancia para un rápido tratamiento.

$\mathrm{Al}$ haber pocos datos epidemiológicos sobre envenenamiento por Loxosceles sp. en animales de compañía, el reporte de casos ayudaría a brindar información clínica y científica para usarse en tratamientos con este tipo de accidente. A pesar de ello, no hay acuerdos comunes respecto a tratamientos terapéuticos para los cuadros de envenenamiento.

\section{Descripción del caso}

Se presenta al servicio de Guardia del Hospital Escuela de Animales Pequeños de la Facultad de Ciencias Veterinarias UNLPam un paciente canino, de 6 años, $7 \mathrm{~kg}$ de peso, hembra entera, raza Fox Terrier, remitida a dicho servicio luego de ser atendida por otros colegas por una supuesta picadura de insecto. A la consulta llegó con un tratamiento sintomático que consistió en dexametasona, un colirio con flurbiprofeno y un coagulante con componentes de ciclonamina y vitamina $\mathrm{K}$ cuyas dosis y vías se desconocen. Sin embargo, a la exploración clínica se obtienen datos de frecuencia cardíaca de 140 lat/min y taquipneica, temperatura de $38,7^{\circ} \mathrm{C}$, linfonódulos explorables sin alteraciones. Se observan lesiones evidentes a la inspección en la cabeza: conjuntiva ocular izquierda muy hiperémica, con sangrado evidente (Foto 2), labio superior del mismo lado con franca edematización y hematoma también con sangrado (Foto 3), edema y hematomas en miembros posteriores en la región poplítea y del muslo. Se realiza interconsulta con el equipo del Área de Identificación y Reconocimiento de Animales Ponzoñosos de la Facultad de Ciencias Veterinarias de la UNLPam, quienes ampliaron la anamnesis a fin de determinar supuesto caso de loxoscelismo.

Como primer método complementario se indica hemograma y bioquímica sanguínea de rutina, resultando un hematocrito de $29 \%$, hemoglobina de 9,67 g/dl. El índice de producción reticulocitaria arrojó un valor de 2,5, lo que refiere un proceso regenerativo, el cual se condice con el frotis sanguíneo que muestra signos de anisocitosis marcada. Tanto la bioquímica hepática como la renal fueron normales. Con estos datos, se decide un protocolo de medicación que consistió, previa colocación de una vía endovenosa y administración de solución $(\mathrm{NaCl}$ al $0,9 \%$ ), en la aplicación de dexametasona ( $1 \mathrm{mg} / \mathrm{kg})$. Pasadas las $3 \mathrm{~h}$, se decide administrar una ampolla de antiveneno loxoscélico (antídoto Loxosceles lote 1015. Vto 31/08/2019. ANLIS “Dr. Carlos G. Malbrán”), de la siguiente manera: $1 \mathrm{ml}$ en forma de bolo lento y a los 20 minutos 
los $4 \mathrm{ml}$ restantes diluidos en dextrosa al 5\% durante las siguientes $3 \mathrm{~h}$ con un protocolo antibiótico que incluyó metronidazol $(25 \mathrm{mg} /$ kg cada $12 \mathrm{~h}$ ) y amoxicilina con ácido clavulánico (12.5 mg/kg). Cabe aclarar que no existe un tratamiento específico con suero antiloxoscélico disponible en Medicina Veterinaria, y que en este caso la ampolla utilizada, próxima a su vencimiento y devolución, fue donada por la dirección de Epidemiología del Ministerio de Salud de La Pampa.

Al día siguiente se repite el hemograma y se agrega un coagulograma, obteniéndose un hematocrito de $23 \%$, con una prueba de coagulación KPTT (Kaolin activated Partial Tromboplastin Time) mayor a 120 segundos y una concentración de protrombina de menos de $10 \%$, por lo que se decide transfundir con sangre entera fresca. Se agrega al protocolo medicamentoso vitamina $\mathrm{K} 1(1 \mathrm{mg} / \mathrm{kg})$ y tramadol $(2 \mathrm{mg} /$ kg cada $12 \mathrm{~h}$ ).

Después de $24 \mathrm{~h}$ las lesiones muestran una notable mejoría, una regresión en su aspecto sangrante y recuperación del estado general del paciente. En los días posteriores los valores de laboratorio se normalizaron: hematocrito: $30 \% 48$ h post transfusión y $45 \% 96$ h post transfusión; y tiempos de coagulación: KPTT 30 segundos y 20 segundos, respectivamente.

Finalmente, se incorpora al protocolo prednisolona en una dosis de $1 \mathrm{mg} / \mathrm{kg}$ cada $24 \mathrm{~h}$ por una semana, y luego se disminuye a $0,5 \mathrm{mg} / \mathrm{kg}$ cada $24 \mathrm{~h}$ por una semana más. El control de laboratorio una semana post episodio arrojó un hematocrito de $41 \%$ con un tiempo de coagulación KPTT de 23 segundos, sin signos clínicos de hemorragias o hematomas.

\section{Conclusiones}

Los accidentes causados por arañas del género Loxosceles sp. son importantes en la clínica de pequeños animales, aunque no existen datos epidemiológicos sobre estos eventos en mascotas. Dada la gravedad de la lesión y las posibles complicaciones sistémicas, resulta necesario saber más sobre la especie y las consecuencias del envenenamiento ${ }^{(8)}$. En Argentina los accidentes en animales domésticos podrían ocurrir por la picadura de Loxosceles laeta, conocida comúnmente como "araña marrón, violinista o reclusa" ${ }^{9}$.

Determinar un accidente provocado por la picadura de esta araña es un desafío para el Médico Veterinario. El conocimiento de los lugares con mayor prevalencia se convierte en un componente esencial, así como todo el cuadro clínico, al momento de establecer un diagnóstico de aracnoidismo ${ }^{(10)}$. 
En el presente caso, el diagnóstico clínico fue acordado interdisciplinariamente con el equipo del Área de Identificación y Reconocimiento de Animales Ponzoñosos de la Facultad de Ciencias Veterinarias de la UNLPam. Por los datos clínicos y las evidencias de la presencia de Loxosceles sp. en el domicilio (ootecas y mudas en un tocadiscos antiguo llevado en esos días para restaurar), se conjetura que el envenenamiento canino sería un caso de loxoscelismo dermo-hemolítico. La utilización del antiveneno, a pesar de que en la literatura hay controversias en el tiempo de aplicación del mismo post accidente, fue un desafío para el grupo de profesionales. Su utilidad estaría relacionada con la indicación de la dosis adecuada y su administración en el momento oportuno para poder evitar o limitar el cuadro necrótico y/o eliminar o menguar la hemólisis en los casos sistémicos. Los antivenenos han demostrado ser eficaces cuando se administran dentro de las primeras $24 \mathrm{~h}$ a $36 \mathrm{~h}$ de ocurrida las picaduras. En los casos cutáneo-viscerales deben aplicarse siempre, aún transcurridas más de 36 horas desde la picadura ${ }^{(5)}$. En Brasil se afirma que la eficacia del tratamiento se ve reducida si éste se realiza después de las 36 horas posteriores al accidente ${ }^{(2)}$.Según las pruebas de laboratorio, el paciente presentó anemia hemolítica aguda, con coagulopatía adquirida. Esto se suma a que la perra había tenido un sangrado muy prolongado en su último estro, dos meses atrás, lo que llevó a pensar en alguna enfermedad metabólica, endócrina, o inmunomediada de base, que pudiese haber agravado el cuadro o incluso algún déficit de factores de la coagulación que se hayan corregido con el tratamiento. La decisión en la administración de sangre entera fue basada en la necesidad de atender la coagulopatía y anemia resultante. Según Couto ${ }^{(11)}$, como norma general, no debe dejar de realizarse una transfusión si se trata de un procedimiento que puede salvar la vida del paciente. El pronóstico fue favorable para las condiciones cutáneas sintomáticas, a pesar de la gravedad de la lesión.

Es necesario difundir los conocimientos básicos para que el loxoscelismo sea un diagnóstico diferencial ante un cuadro compatible, y para que pueda diagnosticarse de manera temprana a partir de los datos clínicos y la anamnesis, con la identificación del animal causante del accidente cuando fuese posible.

Hasta la fecha, no hay acuerdo en la aplicación de un tratamiento común a animales por envenenamiento con toxina de Loxosceles sp. En el presente reporte no se descartó ninguna posibilidad de causas que estuvieran provocando el cuadro hematológico, y desde un inicio fue un tratamiento multifactorial e interdisciplinario con resultados beneficiosos. 


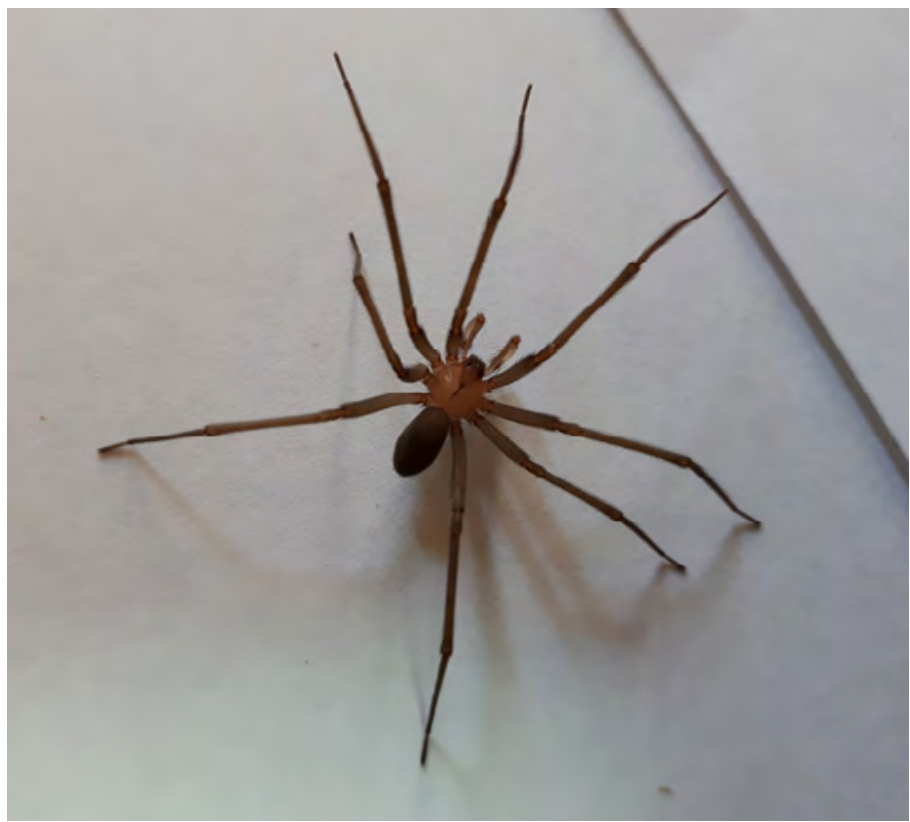

Foto 1. Loxosceles laeta.

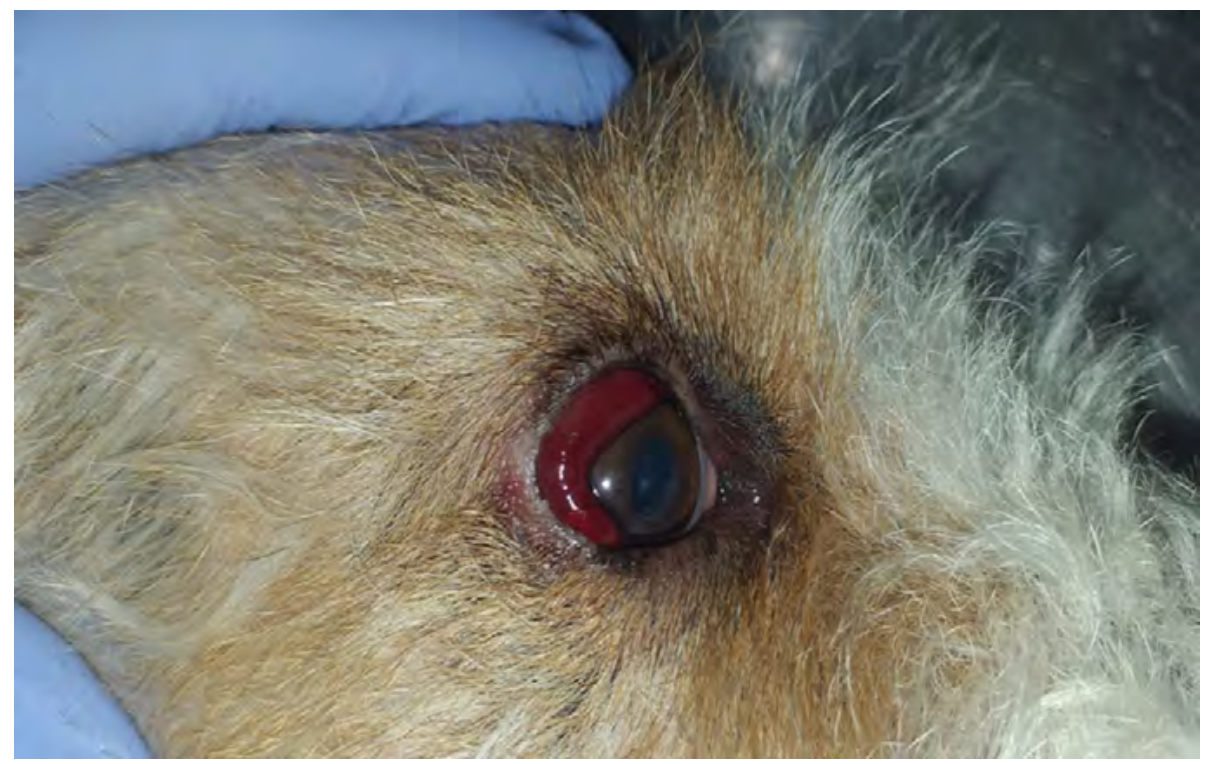

Foto 2. Conjuntiva ocular izquierda muy hiperémica, con sangrado. 


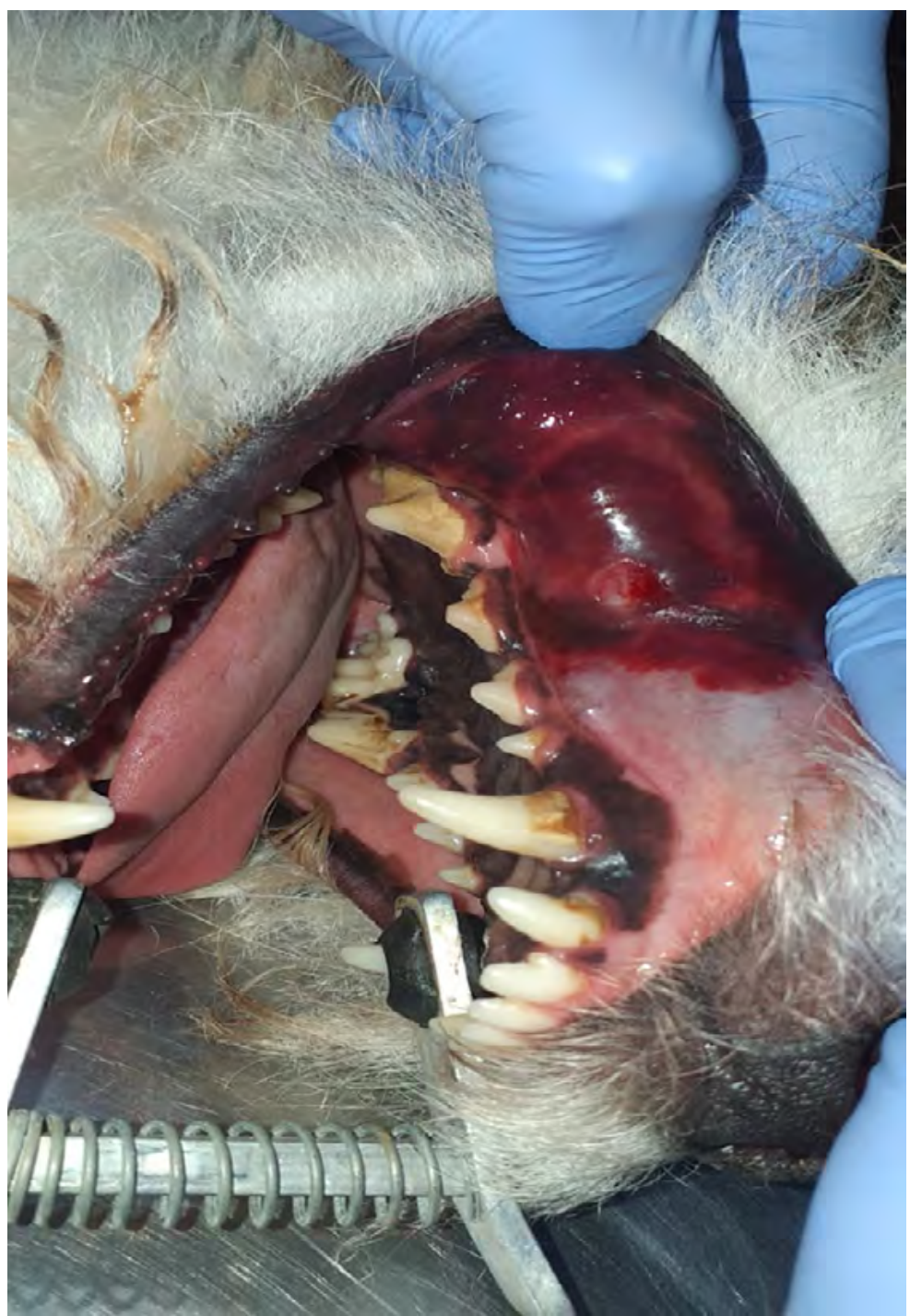

Foto 3. Labio superior izquierdo edematización, hematoma y sangrado. 


\section{Bibliografía}

1. Catálogo de Arañas de Argentina. Museo Argentino de Ciencias Naturales "Bernardino Rivadavia", 2021 en línea en https://sites.google.com/site/catalogodearanasdeargentina/, consultado el 12 de Mayo de 2021.

2. de Roodt A, Salomon O, LLoveras S, Orduna T. Envenenamiento por arañas del género Loxosceles. Medicina (Buenos Aires) 2002; 62 (1): 83-94.

3. Camplesi AC, Albernaz SS, Burger KP, Fredrichsen Moya-Araujo C. Accidents Caused by Spider Bites. Open Journal of Animal Sciences, 2014; 4 (3): 113-117.

4. Bruni M, Marega NA, Ruiz CH. Guía para el reconocimiento y prevención de accidentes por animales ponzoñosos en la región norte de la provincia de La Pampa. Santa Rosa, La Pampa: EdUNLPam; 2020.

5. Instructivo de uso antiveneno loxoscelico (Loxosceles laeta). Instituto Nacional de producción de Biológicos. ANLIS “Dr. Carlos G. Malbrán. 600-IAV-005-00.

6. Ministerio de Salud de la Nación. Guía de prevención, diagnóstico, tratamiento y vigilancia epidemiológica de los envenenamientos por arañas. Buenos Aires, Argentina: Ministerio de Salud de la Nación Argentina; 2012.

7. Guevara, MA, Oviedo R. Loxoscelismo: cuadro cutáneo-víscero-hemolítico en canino. Vet. Arg. 2013; Vol. XXX (No 308).

8. Duarte KO, Ballardin L, Vieira NT, Terra AL. Lesão dermonecrótica em um gato atribuída a envenenamento por Loxosceles - Relato de caso. Ars Veterinaria, Jaboticabal, Brasil 2018; .34 (2): 083-087.

9. Gimenez Raush D, Zeinstger, P. Envenenamiento compatible con picadura de "araña marrón" (Loxosceles laeta) en un canino. En XXXII Jornadas Interdisciplinarias de Toxicología Chubut; 2014.

10. Collacico K, Chanquetti, AM, Ferrari R. Acidente por loxosceles em cão - Relato de caso. Ensaios e Ciência: Ciências Biológicas, Agrárias e da Saúde. 2008; XII (2):179-195.

11. Nelson R, Couto G. Capítulo 83. Anemia. En Medicina interna de pequeños animales. Cuarta edición. Elseiver. 2010. 